\title{
Analysis of treatment patterns and persistence on branded and generic medications in major depressive disorder using retrospective claims data
}

This article was published in the following Dove Press journal:

Neuropsychiatric Disease and Treatment

25 October 2016

Number of times this article has been viewed

\author{
Caitlyn T Solem' \\ Ahmed Shelbaya ${ }^{2,3}$ \\ Yin Wan' \\ Chinmay G Deshpande' \\ Jose Alvir ${ }^{2}$ \\ Elizabeth Pappadopulos ${ }^{2}$ \\ 'Pharmerit International, Real \\ World Evidence/Data Analytics, \\ Bethesda, MD, ${ }^{2}$ Pfizer, Inc., Global \\ Health Outcomes, New York, NY, \\ ${ }^{3}$ Epidemiology Department of \\ Mailman's School of Public Health, \\ Columbia University Mailman Schoo \\ of Public Health, New York, NY, USA
}

Correspondence: Caitlyn T Solem Pharmerit International, Real World Evidence/Data Analytics, 4350 East-West Hwy, Suite \#430, Bethesda, MD 208I4, USA

$\mathrm{Tel}+|24082| 1267$

Fax $+\mid 2408211296$

Email csolem@pharmerit.com
Background: In major depressive disorder (MDD), treatment persistence is critical to optimize symptom remission, functional recovery, and health care costs. Desvenlafaxine tends to have fewer drug interactions and better tolerability than other MDD drugs; however, its use has not been assessed in the real world.

Objective: The aim of the present study is to compare medication persistence and concomitant MDD drug use with branded desvenlafaxine (Pristiq ${ }^{\circledR}$ ) compared with antidepressant drug groups classified as 1) branded selective serotonin reuptake inhibitors (SSRIs; ie, escitalopram $\left[\right.$ Lexapro $\left.^{\mathrm{TM}}\right]$ ) and selective serotonin-norepinephrine reuptake inhibitors (SNRIs; ie, venlafaxine $\left[\right.$ Effexor $\left.^{\circledR}\right]$, duloxetine $\left[\right.$ Cymbalta $\left.^{\circledR}\right]$ ) and 2) generic SSRIs/SNRIs (ie, escitalopram, citalopram, venlafaxine, fluvoxamine, fluoxetine, sertraline, paroxetine, and duloxetine).

Patients and methods: MDD patients (ICD-9-CM codes 296.2, 296.3), with $\geq 2$ prescription fills for study drugs and 12-month preindex continuous enrollment from the MarketScan Commercial Claims and Encounters Database (2009-2013), were included. Time-to-treatment discontinuation (prescription gap $\geq 45$ days) was assessed using the Kaplan-Meier curve and Cox model. Concomitant MDD drug use was compared.

Results: Of the 273,514 patients included, 14,379 patients were initiated with branded desvenlafaxine, 50,937 patients with other branded SSRIs/SNRIs, and 208,198 patients with generic SSRIs/SNRIs. The number of weeks for treatment discontinuation for branded desvenlafaxine were longer (40.7 [95\% CI: 39.3, 42.0]) compared with other branded SSRIs/ SNRIs (28.9 [95\% CI: 28.4, 29.1]) and generic SSRIs/SNRIs (33.4 [95\% CI: 33.1, 33.7]). Adjusting for baseline characteristics, patients who were prescribed with other branded SSRIs/SNRIs were $31 \%$ and generic SSRIs/SNRIs were $11 \%$ more likely to discontinue treatment compared with branded desvenlafaxine. In sensitivity analysis, the risk of discontinuation was within $10 \%$ of branded desvenlafaxine for branded duloxetine, generic escitalopram, and generic venlafaxine. Concomitant MDD drug use was higher among branded desvenlafaxine patients (43.8\%) compared with other branded SSRIs/SNRIs (39.8\%) and generic SSRIs/SNRIs (36.4\%).

Conclusion: MDD patients on branded desvenlafaxine were more persistent with treatment compared with those on other branded or generic SSRI/SNRI therapies. Future research should include assessments of underlying factors on the treatment persistence in MDD patients.

Keywords: selective serotonin reuptake inhibitors, selective serotonin-norepinephrine re-uptake inhibitors

\section{Background}

Major depressive disorder (MDD) is one of the leading causes of disability in people 15-44 years old. ${ }^{1}$ The point prevalence of MDD globally was estimated to be 298 million people or $4.7 \%$ of the population. ${ }^{2}$ Lifetime prevalence estimates vary 
across countries ranging from $3 \%$ in Japan to $17 \%$ in the US. $^{3}$ In 2013, it was estimated that 15.7 million people of age 18 years or older in the US had at least one major depressive episode in the past year. This accounts for $6.7 \%$ of all US adults. The total economic burden of depressive disorder in the US was estimated to be US\$83.1 billion in 2000, of which US\$26.1 billion (31\%) accounted for direct medical costs, US\$51.5 billion (62\%) were workplace costs, and US\$5.4 billion (7\%) were suicide-related mortality costs. ${ }^{4}$

People with MDD are undertreated. Only 50\% of those diagnosed with MDD receive any treatment in a given year. ${ }^{5,6}$ Recently, most MDD patients are treated with pharmacotherapy, psychotherapy, or their combination. First-line pharmacotherapy options include the selective serotonin reuptake inhibitors (SSRIs) and the selective serotoninnorepinephrine reuptake inhibitors (SNRIs). ${ }^{7}$ Second-line pharmacotherapy options include the monoamine oxidase inhibitors (MAOIs) and the tricyclic antidepressants (TCAs). ${ }^{7}$ Among the treated patients, concomitant medication use with other antidepressants or antipsychotics is common. In a retrospective study using Texas Medicaid data, 35\% of patients treated with an SSRI also received concomitant antianxiety or sleep medications. ${ }^{8}$ Based on a meta-analysis of ten clinical trials, there is an evidence that the adjunct use of antipsychotics with antidepressant treatment might lead to better remission compared with the adjunct use of placebo. ${ }^{9}$ The effect of concomitant medication use of antipsychotics, other antidepressants including SSRI/SNRIs, and TCAs on MDD treatment persistence remains unclear.

Patients with MDD are three times more likely to be nonadherent than nondepressed individuals. ${ }^{10}$ Improved persistence to antidepressant medication may lead to prolonged depression remissions, increased work productivity, and reduced overall costs. ${ }^{11}$ Well-known factors contributing to poor antidepressant therapy persistence include adverse events and lack of efficacy. ${ }^{12}$ Suboptimal treatment outcomes due to nonpersistence, premature medication discontinuation, and antidepressant medication switching are consistently associated with higher health care costs. ${ }^{13}$

There is limited published literature focusing on drug class comparisons with respect to outcomes in MDD including adherence and use of concomitant medication. Pristiq ${ }^{\circledR}$, O-desmethylvenlafaxine (also referred to as desvenlafaxine; Pfizer, Inc., New York, NY, USA), a main metabolite of Effexor ${ }^{\circledR}$ (venlafaxine hydrochloride), is an SNRI approved by the US FDA in 2008. Desvenlafaxine tends to have a lower propensity for drug interactions and a better tolerability profile among other antidepressants allowing easier coadministration with other medications due to the weak inhibitory effects it has on the hepatic Cytochrome P450 (CYP) system. ${ }^{14}$ In real-world settings, there is a need to understand if desvenlafaxine's better tolerability profile translates to improve adherence and concomitant drug use.

The objective of this study was to examine antidepressant persistence and use of concomitant medications (other SNRIs/SSRIs or nonstudy drugs including TCAs, MAOIs, antipsychotics, and atypical antidepressants) among patients receiving branded desvenlafaxine (Pristiq; Pfizer, Inc.) compared with 1) other branded SSRIs/SNRIs (venlafaxine [Effexor; Pfizer, Inc.], duloxetine [Cymbalta ${ }^{\circledR}$, Eli Lilly and Company, Indianapolis, IN, USA], and escitalopram [Lexapro ${ }^{\text {TM }}$, Forest Laboratories, New York, NY, USA]) and 2) generic SSRIs/SNRIs (venlafaxine, escitalopram, fluvoxamine, sertraline, citalopram, fluoxetine and paroxetine) using real-world database.

\section{Patients and methods \\ Data source}

Data on patients with MDD were extracted from the Truven Health Analytics MarketScan Commercial Claims and Encounters Database from January 1, 2009, to December 31, 2013. The MarketScan Commercial Claims and Encounters Database is a high-quality resource with the combined claims of $\sim 100$ employers and 12 US health plans representing $>30$ million covered lives including employees and their adult dependents. The statistical analyses utilized de-identified secondary data source (Truven Health Analytics MarketScan database). No institutional review board approval was required as the statistical analyses utilized deidentified secondary data source (Truven Health Analytics MarketScan database).

\section{Sample selection and study drugs}

Adult ( $\geq 18$ years) patients with at least one inpatient or outpatient claim for MDD (ICD-9-CM codes 296.2 and 296.3) were identified. Patients diagnosed with schizophrenia (ICD-9 CM: 295.x) or bipolar disorder (296.0, 296.1, 296.4-7, 296.80, 296.81, and 296.89) were excluded. The National Drug Codes from RED BOOK ${ }^{\mathrm{TM}}$ were used to identify the brand and generic study drugs of interest (Table 1). In order to be included in the cohort for a particular study drug, patients needed at least two prescription claims for that drug, with the date of first fill serving as the index date. Patients were additionally required to have continuous enrollment for at least 12 months before (baseline period) and 6 months after the index date and at least one inpatient or outpatient claim for MDD within the preindex period of 12 months or within 30 days after the index date. 
Table I List of study drugs and classes

\begin{tabular}{|c|c|c|}
\hline Study drug & $\begin{array}{l}\text { Therapeutic } \\
\text { class }\end{array}$ & $\begin{array}{l}\text { Antidepressant } \\
\text { drug class }\end{array}$ \\
\hline Pristiq (desvenlafaxine) & SNRIs & Branded desvenlafaxine \\
\hline $\begin{array}{l}\text { Cymbalta } \\
\text { (branded duloxetine) }\end{array}$ & SNRIs & Other branded SSRIs/SNRIs \\
\hline $\begin{array}{l}\text { Lexapro } \\
\text { (branded escitalopram) }\end{array}$ & SSRIs & Other branded SSRIs/SNRIs \\
\hline $\begin{array}{l}\text { Effexor } \\
\text { (branded venlafaxine) }\end{array}$ & SNRIs & Other branded SSRIs/SNRIs \\
\hline Generic fluoxetine & SSRIs & Generic SSRIs/SNRIs \\
\hline Generic fluvoxamine & SSRIs & Generic SSRIs/SNRIs \\
\hline Generic escitalopram & SSRIs & Generic SSRIs/SNRIs \\
\hline Generic venlafaxine & SNRIs & Generic SSRIs/SNRIs \\
\hline Generic citalopram & SSRIs & Generic SSRIs/SNRIs \\
\hline Generic sertraline & SSRIs & Generic SSRIs/SNRIs \\
\hline Generic paroxetine & SSRIs & Generic SSRIs/SNRIs \\
\hline Generic duloxetine & SNRIs & Generic SSRIs/SNRIs \\
\hline
\end{tabular}

Abbreviations: SNRIs, selective norepinephrine reuptake inhibitors; SSRIs, selective serotonin reuptake inhibitors.

Once patients were identified, study drug cohorts were grouped into the following larger categories for primary comparisons: 1) branded desvenlafaxine, 2) other branded SSRIs/SNRIs (Cymbalta, Effexor, and Lexapro), and 3) generic SSRIs/SNRIs (venlafaxine, escitalopram, paroxetine, sertraline, citalopram, fluoxetine and fluvoxamine; Table 1). There were no patients with generic duloxetine in the data set given that a generic version was not available in 2013. The rationale for selection of branded study drugs was based on specific SSRIs and SNRIs that had a generic version on the market at the time of the study; however, we also considered other drugs including Viibryd (vilazodone), Fetzima (levomilnacipran), and Brintellix (vortioxetine). These drugs were not included due to smaller sample sizes (launched in/after 2011). Individual drugs were also compared with branded desvenlafaxine in the sensitivity analyses.

\section{Outcomes}

Persistence was measured by calculating the time from index date to treatment discontinuation. Patients were persistent if they received continuous treatment with the same study drug without a prescription gap of $\geq 45$ days from the index date to the end of continuous enrollment or the end of the study period. Patients were nonpersistent if they discontinued the study drug, with or without switching to another antidepressant (ie, no prescription claim for the index study drug in the 45 days following the end of the index study drug supply). Nonpersistent patients were also followed until the end of patient continuous enrollment or the end of the study period.

Concomitant antidepressant drug use was defined as a prescription claim for another branded or generic SNRI/
SSRI or a nonstudy drug (TCAs, MAOIs, antipsychotics, and atypical antidepressants) between the index date and the last fill date for the index study drug. Concomitant antidepressant drugs included were study drugs (branded desvenlafaxine, branded SNRIs, generic SNRIs, generic SSRIs, and branded SSRIs), nonstudy branded SSRIs (ie, Celexa ${ }^{\mathrm{TM}}$ [citalopram hydrobromide; Forest Laboratories], Luvox ${ }^{\circledR}$ [fluvoxamine maleate; Solvay Pharmaceuticals, Inc., Marietta, GA, USA], Zoloft $^{\circledR}$ [sertraline hydrochloride; Pfizer, Inc.], Prozac ${ }^{\circledR}$ [fluoxetine, Eli Lilly and Company], Sarafem ${ }^{\circledR}$ [fluoxetine hydrochloride; Eli Lilly and Company], Paxil ${ }^{\circledR}$ [paroxetine; GlaxoSmithKline plc, London, UK], Brisdelle ${ }^{\circledR}$ [paroxetine; Noven Therapeutics, LLC, Miami, FL, USA], Pexeva ${ }^{\circledR}$ [paroxetine; Noven Therapeutics, LLC]), TCAs, MAOIs, antipsychotics, and atypical antidepressants.

\section{Statistical analysis}

Descriptive statistics were used to compare baseline demographic characteristics among the three drug larger categories (ie, branded desvenlafaxine, other branded SSRIs/SNRIs, and generic SSRIs/SNRIs). The principal variables of interest were concomitant drug use and persistence. Concomitant drug use among the three drug categories was also examined. The proportion of patients with any concomitant antidepressant drug use was calculated; the proportion of patients receiving monotherapy or combination concomitant drug therapy in MDD was explored and compared across the three drug categories.

For the persistence analysis, nonparametric KaplanMeier curves were used to estimate the time to discontinuation (defined as events) controlling for data censoring at the end of data cut (administrative censoring) or loss of continuous enrollment and were compared among the three drug categories and by specific drugs. In addition, persistence was examined using a semiparametric Cox model to adjust for baseline characteristics that may have differed between drug comparison groups. Age, sex, insurance, region, preindex Charlson comorbidity index, prior hospital admissions, psychiatric comorbidities (including generalized anxiety disorder, posttraumatic stress disorder (PTSD), dementia, and personality disorder), and preindex MDD costs were included as covariates. Model selection was based on a combination of prespecified clinically relevant covariates regardless of significance (ie, sex, region, insurance, and psychiatric comorbidities) and backward selection (significance level of 0.05 ) for the remaining variables. Additionally, a sensitivity analysis was performed to examine and compare the time to discontinuation and persistence (using Cox model) among all individual SSRI/SNRI study drugs. 
For all analyses, a two-sided alpha of 0.05 was used for significance testing. The analysis was performed using SAS 9.4 (SAS Institute Inc., Cary, NC, USA).

\section{Results}

\section{Study population}

A total of 273,514 patients met the inclusion and exclusion criteria, of which 14,379 patients were initiated with branded desvenlafaxine, 50,937 patients with other branded SSRIs/SNRIs, and 208,198 patients with generic SSRIs/ SNRIs (Figure 1). Mean age across drug cohorts ranged from 46.2 years (branded desvenlafaxine) to 47.7 years (other branded SSRIs/SNRIs). Percentage of females across the cohorts ranged from 70.1 (generic SSRIs/SNRIs) to 73.8 (branded desvenlafaxine; Table 2). Most branded desvenlafaxine users resided in the South (48.5\%) and had a preferred provider organization (PPO) insurance plan (64\%). Comorbidities and hospital admissions for patients who were prescribed with branded desvenlafaxine were lower compared with the other branded/generic SSRIs/SNRIs drug categories.

\section{Persistence to antidepressant drugs}

The median time to treatment discontinuation (using KaplanMeier analysis) for branded desvenlafaxine was 40.7 weeks (95\% CI: 39.3-42.0), which was significantly longer compared with other branded SSRIs/SNRIs (28.9 weeks [95\% CI: 28.4, 29.1]) and generic SSRIs/SNRIs (33.4 weeks

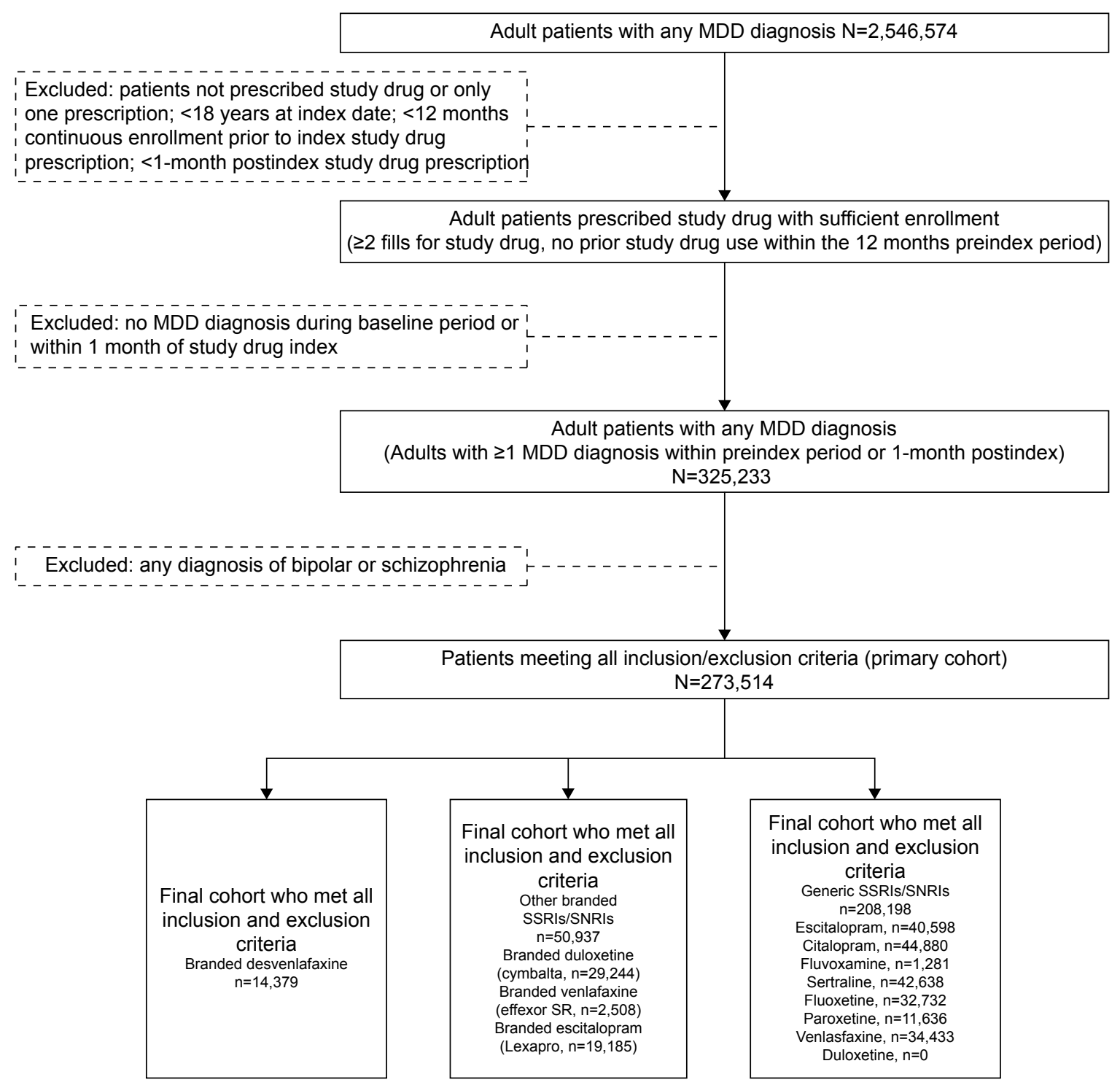

Figure I Flowchart for cohort selection.

Abbreviations: MDD, major depressive disorder; SSRIs, selective serotonin reuptake inhibitors; SNRIs, selective norepinephrine reuptake inhibitors. 
Table 2 Preindex demographic characteristics by drug class ${ }^{\mathrm{a}}$

\begin{tabular}{|c|c|c|c|}
\hline Characteristics & $\begin{array}{l}\text { Branded desvenlafaxine } \\
(\mathrm{N}=\mid 4,379)\end{array}$ & $\begin{array}{l}\text { Other branded SSRIs/SNRIs } \\
(\mathbf{N}=\mathbf{5 0 , 9 3 7 )}\end{array}$ & $\begin{array}{l}\text { Generic SSRIs/SNRIs } \\
(\mathbf{N}=\mathbf{2 0 8}, 198)\end{array}$ \\
\hline Age, mean years (SD) & $46.2(12.6)$ & $47.7(14.2)$ & $47(14.9)$ \\
\hline Female, n (\%) & $10,616(73.8)$ & $36,286(71.2)$ & $145,869(70.1)$ \\
\hline \multicolumn{4}{|l|}{ Region, n (\%) } \\
\hline Northeast & $\mathrm{I}, 50 \mathrm{I}(\mathrm{I} 0.4)$ & $7,527(\mid 4.8)$ & $31,248(15)$ \\
\hline North-central & $3,168(22.0)$ & I3, I $67(25.8)$ & $52,510(25.2)$ \\
\hline South & $6,976(48.5)$ & 19,244 (37.8) & $67,176(32.3)$ \\
\hline West & $2,597(18.1)$ & $10,454(20.5)$ & $55,480(26.6)$ \\
\hline Unknown & $137(1.0)$ & $545(I . I)$ & I,784 (0.9) \\
\hline \multicolumn{4}{|l|}{ Insurance type, n (\%) } \\
\hline Comprehensive & $553(3.8)$ & $3,024(5.9)$ & II,39| (5.5) \\
\hline HMO & I,952 (I3.6) & $7,669(15.1)$ & 44,705 (2I.5) \\
\hline Others & $1,014(7.1)$ & $3,430(6.7)$ & $13,860(6.7)$ \\
\hline POS & $1,288(9.0)$ & $4,112(8.1)$ & $15,738(7.6)$ \\
\hline PPO & $9,234(64.2)$ & $31,362(61.6)$ & II5,6I3 (55.5) \\
\hline Missing & $338(2.4)$ & $\mathrm{I}, 340(2.6)$ & 6,89I (3.3) \\
\hline \multicolumn{4}{|l|}{ Charlson comorbidity index, n (\%) } \\
\hline 0 & $8,993(62.5)$ & $29,749(58.4)$ & $124,889(60)$ \\
\hline 1 & $2,973(20.7)$ & $|0,25|(20.1)$ & $41,746(20.1)$ \\
\hline 2 & $\mathrm{I}, 284(8.9)$ & $5,048(9.9)$ & $19,202(9.2)$ \\
\hline$\geq 3$ & I, I29 (7.9) & $5,889(11.6)$ & $22,36 \mathrm{I}(10.7)$ \\
\hline \multicolumn{4}{|l|}{ Psychiatric comorbidities, n (\%) } \\
\hline Generalized anxiety disorder & $2,288(I 5.9)$ & $7,573(\mid 4.9)$ & $28,555(13.7)$ \\
\hline Posttraumatic stress disorder & $527(3.7)$ & $1,790(3.5)$ & $6,936(3.3)$ \\
\hline Dementia & $57(0.4)$ & $558(1.1)$ & $2,217(1.1)$ \\
\hline Substance abuse disorder & I,853 (I2.9) & $7,086(13.9)$ & $29,514(14.2)$ \\
\hline Personality disorder & $188(1.3)$ & $700(1.4)$ & $2,749(1.3)$ \\
\hline Premenstrual dysphoric disorder & $182(1.3)$ & $543(1.1)$ & $2,020(1.0)$ \\
\hline \multicolumn{4}{|l|}{ Hospital admissions, n (\%) } \\
\hline 1 & I,70I (II.8) & $7,759(15.2)$ & $28,768(13.8)$ \\
\hline 2 & $390(2.7)$ & $1,907(3.7)$ & $6,699(3.2)$ \\
\hline $3+$ & $195(1.4)$ & $1,169(2.3)$ & $3,662(1.8)$ \\
\hline $\begin{array}{l}\text { Preindex MDD-related drug costs } \\
\geq \$ 1,000, n(\%)\end{array}$ & $4,342(30.2)$ & $9,580(18.8)$ & $52,068(25.0)$ \\
\hline Pre-index drug costs $\geq \$ 1,000, \mathrm{n}(\%)$ & $9,414(65.5)$ & $30,325(59.5)$ & II 5,766 (55.6) \\
\hline
\end{tabular}

Note: ${ }^{a}$ Data presented as number of patients (\%).

Abbreviations: SSRI, selective serotonin reuptake inhibitor; SNRI, serotonin-norepinephrine reuptake inhibitor; HMO, Health Maintenance Organization; POS, point of service; PPO, preferred provider organization; MDD, major depressive disorder.

[95\% CI: 33.1, 33.7]). Upon comparing the individual drugs, the median time to discontinuation was shortest for branded venlafaxine hydrochloride ( 18.7 weeks [95\% CI: $18.1,19.9]$ ) and longest for generic escitalopram (50.4 weeks [95\% CI: $49.3,51.6])$. Overall, the median time to discontinuation was longer for branded desvenlafaxine compared with other branded SSRIs/SNRIs and generic SSRIs/SNRIs as a drug category and all individual study drugs (SSRIs/SNRIs), with the exception of generic escitalopram (median time to discontinuation of 50.4 weeks [ $95 \% \mathrm{CI}: 49.3,51.6])$ and generic venlafaxine (median time to discontinuation of 45.4 weeks [95\% CI: 44, 46.7]).

At 6 months, $60.4 \%$ (95\% CI: 59.6, 61.2) of patients treated with branded desvenlafaxine remained persistent; at 12 months, $42.5 \%$ (95\% CI: $41.7,43.4)$ of patients treated with branded desvenlafaxine remained persistent. Persistence estimates for branded desvenlafaxine users at 6 months and 12 months were significantly higher compared with other branded and generic SSRI/SNRI drug categories (Table 3). At 6 months, persistence for branded desvenlafaxine was higher than most other individual drugs (eg, all individual branded SSRIs/SNRIs, generic citalopram, fluvoxamine, sertraline, fluoxetine, and paroxetine); it was similar to generic venlafaxine and marginally lower than generic escitalopram.

After adjusting for baseline demographic and clinical characteristics using the Cox proportional model, patients treated with other branded SSRI/SNRI (hazard ratio [HR] 1.31 [95\% CI: 1.28, 1.34], $P<0.001)$ or generic SSRI/SNRI (HR 1.11 [95\% CI: 1.08, 1.13], $P<0.001$ ) drug categories 
Table 3 Persistence by antidepressant treatment ${ }^{\mathrm{a}}$

\begin{tabular}{|c|c|c|c|}
\hline \multirow[t]{2}{*}{ Antidepressant } & \multirow{2}{*}{$\begin{array}{l}\text { Median weeks to } \\
\text { discontinuation }(95 \% \mathrm{Cl})^{\mathrm{b}}\end{array}$} & \multicolumn{2}{|c|}{ Persistent patients $\%(95 \% \mathrm{Cl})$} \\
\hline & & 6 months & 12 months \\
\hline \multicolumn{4}{|l|}{ Study drug categories } \\
\hline Branded desvenlafaxine & $40.7(39.3-42.0)$ & $60.4(59.6-61.2)$ & $42.5(4 \mid .7-43.4)$ \\
\hline Other branded SSRIs/SNRIs & $28.9(28.4-29.1)$ & $51.9(51.5-52.3)$ & $31.6(31.2-32.1)$ \\
\hline Generic SSRIs/SNRIs & $33.4(33.1-33.7)$ & $55.2(55.0-55.5)$ & $38.6(38.4-38.8)$ \\
\hline \multicolumn{4}{|l|}{ Individual drug categories } \\
\hline Branded desvenlafaxine & $40.7(39.3-42.0)$ & $60.4(59.6-61.2)$ & $42.5(4 \mid .7-43.4)$ \\
\hline Branded duloxetine (Cymbalta) & $35.6(34.9-36.4)$ & 57.1 (56.6-57.7) & $39.6(39.0-40.2)$ \\
\hline Branded venlafaxine (Effexor) & $18.7(\mid 8.1-19.9)$ & $28.2(26.4-29.9)$ & $10.5(9.3-11.8)$ \\
\hline Branded escitalopram (Lexapro) & $25.1(24.6-25.6)$ & $47.0(46.3-47.7)$ & $22.8(22.2-23.5)$ \\
\hline Citalopram & $27.0(26.6-27.4)$ & $49.9(49.5-50.4)$ & $32.1(31.6-32.5)$ \\
\hline Escitalopram & $50.4(49.3-51.6)$ & $63.8(63.3-64.2)$ & $48.7(48.2-49.3)$ \\
\hline Fluoxetine & $28.4(28.0-29.0)$ & $51.4(50.9-52.0)$ & $33.7(33 . I-34.2)$ \\
\hline Fluvoxamine & $22.4(20.4-24.9)$ & $45.3(42.5-48.0)$ & $29.8(27.1-32.4)$ \\
\hline Paroxetine & $27.0(26.1-28.0)$ & $49.8(48.9-50.8)$ & $32.6(31.7-33.5)$ \\
\hline Sertraline & $30.3(29.7-30.7)$ & $52.9(52.5-53.4)$ & $35.7(35.2-36.1)$ \\
\hline Venlafaxine & $45.4(44.0-46.7)$ & $60.8(60.3-61.3)$ & $46.3(45.8-46.9)$ \\
\hline
\end{tabular}

Notes: a The data were calculated using Kaplan-Meier analysis. ${ }^{\text {b}} \mathrm{All}$ patients were censored at the end of follow-up. For time to discontinuation, patients who switched to other antidepressants were censored.

Abbreviations: $\mathrm{Cl}$, confidence interval; SSRIs, selective serotonin reuptake inhibitors; SNRIs, selective norepinephrine reuptake inhibitors.

were more likely to discontinue therapy compared with branded desvenlafaxine (Table 4). Other factors identified influencing the time to discontinuation were prior hospital admission and psychiatric disorders including generalized anxiety disorder, PTSD, and substance abuse disorder. When all individual drugs were considered separately in a sensitivity analysis, compared with branded desvenlafaxine, all other individual antidepressants evaluated were more likely to be discontinued with the exception of generic escitalopram (HR 0.90 [95\% CI: 0.88-0.93]) and generic venlafaxine (HR 0.95 [95\% CI: 0.93-0.98]).

\section{Concomitant drugs use}

Forty-four percent of branded desvenlafaxine, $40 \%$ of other branded SSRIs/SNRIs, and 36\% of generic SSRIs/ SNRIs patients were using concomitant drugs. The most frequently used concomitant drugs were atypical antidepressants, followed by antipsychotics and combination atypical antidepressant-antipsychotic therapy. More than $70 \%$ of patients on branded desvenlafaxine used only one drug concomitantly. Multiple combinations $(+2,+3$, or +4 classes of antidepressants) of concomitant medication use were low $(<20 \%$ patients) among antidepressant users (Table 5).

\section{Discussion}

Our real-world cohort study demonstrates that patients who were prescribed with branded desvenlafaxine had significantly higher persistence compared with other branded and generic SSRI/SNRI drug categories. Over one-third of patients were receiving concomitant SSRI/SNRI therapy; concomitant medication use was highest for patients who were prescribed with branded desvenlafaxine.

Grouping these data into other branded and generic SSRI and SNRI categories helped us gain a broader perspective comparing branded desvenlafaxine outcomes (persistence and concomitant use). Although more patients used generic SNRIs/SSRIs and other branded SNRI/SSRI categories compared with branded desvenlafaxine during the study period, patient demographic characteristics were comparable across the antidepressant drug classes. After multivariable adjustment for demographic and clinical characteristics, patients on branded desvenlafaxine were more likely to be persistent compared with other branded and generic SSRI/SNRI antidepressants. According to the Diagnostic and Statistical Manual of Mental Disorders and American Psychiatric Association guideline ${ }^{15}$ and other literature, ${ }^{16}$ longer treatment persistence could lead to better remission rates for depression. Furthermore, the American College of Physicians recommends that patients should remain on the same antidepressant treatment for at least 4-9 months or longer to prevent a relapse. ${ }^{17}$ A study evaluating Medicaid population $(\mathrm{N}=4,032)$ with depression suggests that adherent patients are less likely to experience a relapse or recurrence of a depressive episode. ${ }^{18}$ However, evidence suggests that patients who do not respond to antidepressant therapy may benefit from a therapy change. In a study on an outpatient sample, $25 \%$ of patients $(\mathrm{N}=727)$ experienced improvement in depression symptoms following treatment with venlafaxine 
Table 4 Persistence by study drug classes (adjusted Cox proportional analysis)

\begin{tabular}{|c|c|c|c|c|}
\hline \multirow[t]{2}{*}{ Variable } & \multirow{2}{*}{$\begin{array}{l}\text { Model for drug } \\
\text { categories } \\
\text { HR }(95 \% \mathrm{Cl})^{\mathrm{a}} \\
\end{array}$} & \multirow[t]{2}{*}{$P$-value } & \multirow{2}{*}{$\begin{array}{l}\text { Model for all } \\
\text { individual drugs } \\
\mathrm{HR}(95 \% \mathrm{Cl})^{\mathrm{a}}\end{array}$} & \multirow[t]{2}{*}{$P$-value } \\
\hline & & & & \\
\hline \multicolumn{5}{|c|}{ Antidepressant drug class (ref: branded desvenlafaxine) } \\
\hline Other branded SSRIs/SNRIs & $1.31(1.28-1.34)$ & $<0.001$ & & \\
\hline Generic SSRIs/SNRIs & $1.11(1.08-1.13)$ & $<0.001$ & & \\
\hline Branded duloxetine (Cymbalta) & & & $1.08(1.05-1.11)$ & $<0.001$ \\
\hline Branded venlafaxine (Effexor) & & & $2.33(2.23-2.45)$ & $<0.001$ \\
\hline Branded escitalopram (Lexapro) & & & $1.65(1.61-1.69)$ & $<0.001$ \\
\hline Citalopram & & & $1.26(1.23-1.29)$ & $<0.001$ \\
\hline Escitalopram & & & $0.90(0.88-0.93)$ & $<0.001$ \\
\hline Fluoxetine & & & $1.22(1.19-1.25)$ & $<0.001$ \\
\hline Fluvoxamine & & & $1.42(1.32-1.52)$ & $<0.001$ \\
\hline Paroxetine & & & $1.31(1.28-1.35)$ & $<0.001$ \\
\hline Sertraline & & & $1.15(1.12-1.18)$ & $<0.001$ \\
\hline Venlafaxine & & & $0.95(0.93-0.98)$ & $<0.001$ \\
\hline \multicolumn{5}{|l|}{ Age (ref: 18-44) } \\
\hline $45-64$ years & $0.83(0.82-0.84)$ & $<0.001$ & $0.84(0.83-0.85)$ & $<0.001$ \\
\hline $65+$ years & $0.79(0.77-0.80)$ & $<0.001$ & $0.78(0.77-0.80)$ & $<0.001$ \\
\hline \multicolumn{5}{|l|}{ Charlson comorbidity index (ref: 0) } \\
\hline I & $1.01(1.01-1.02)$ & 0.055 & $1.02(1.01-1.03)$ & 0.002 \\
\hline 2 & $1.00(0.99-1.02)$ & 0.569 & $1.02(1.00-1.04)$ & 0.039 \\
\hline $3+$ & $1.02(1.01-1.03)$ & 0.101 & $1.03(1.02-1.05)$ & $<0.001$ \\
\hline Female (ref: male) & $0.99(0.98-1.00)$ & 0.007 & $0.99(0.98-1.00)$ & 0.094 \\
\hline \multicolumn{5}{|l|}{ Prior hospital admissions (ref: 0) } \\
\hline I & $1.05(1.04-1.07)$ & $<0.001$ & $1.05(1.03-1.06)$ & $<0.001$ \\
\hline 2 & $1.12(1.09-1.15)$ & $<0.001$ & $1.12(1.08-1.15)$ & $<0.001$ \\
\hline $3+$ & $1.19(1.15-1.23)$ & $<0.001$ & $1.20(1.15-1.26)$ & $<0.001$ \\
\hline \multicolumn{5}{|l|}{ Insurance type (ref: POS) } \\
\hline $\mathrm{HMO}$ & $0.96(0.94-0.98)$ & $<0.001$ & $0.95(0.93-0.97)$ & $<0.001$ \\
\hline PPO & $0.96(0.95-0.98)$ & $<0.001$ & $0.96(0.94-0.98)$ & $<0.001$ \\
\hline Comprehensive & $1.03(1.01-1.06)$ & 0.010 & $1.03(1.00-1.06)$ & 0.034 \\
\hline Others & $0.99(0.97-1.02)$ & 0.806 & $0.99(0.97-1.02)$ & 0.671 \\
\hline \multicolumn{5}{|l|}{ Costs } \\
\hline $\begin{array}{l}\$ 1,000+\text { preindex MDD-related drug costs } \\
\text { (ref: }<\$ 1,000)\end{array}$ & $0.72(0.7 I-0.73)$ & $<0.001$ & $0.77(0.76-0.78)$ & $<0.001$ \\
\hline$\$ 1,000+$ preindex drug cost $($ ref: $<\$ 1,000)$ & $0.87(0.86-0.88)$ & $<0.001$ & $0.89(0.88-0.90)$ & $<0.001$ \\
\hline \multicolumn{5}{|l|}{ Psychiatric comorbidities } \\
\hline Generalized anxiety disorder & $0.97(0.96-0.98)$ & $<0.001$ & $0.98(0.97-1.00)$ & 0.024 \\
\hline Dementia & $0.92(0.88-0.97)$ & 0.002 & $0.93(0.88-0.97)$ & 0.002 \\
\hline Posttraumatic stress disorder & $1.11(1.09-1.14)$ & $<0.001$ & $1.12(1.10-1.15)$ & $<0.001$ \\
\hline Personality disorder & $1.04(1.00-1.09)$ & 0.046 & $1.04(1.00-1.08)$ & 0.059 \\
\hline Premenstrual dysphoric disorder & $0.99(0.94-1.04)$ & 0.649 & $0.99(0.94-1.04)$ & 0.620 \\
\hline Substance abuse disorder & $1.12(1.10-1.13)$ & $<0.001$ & $1.12(1.11-1.14)$ & $<0.001$ \\
\hline \multicolumn{5}{|l|}{ Region, n (\%) (ref: Northeast) } \\
\hline North-central & $1.01(0.99-1.02)$ & 0.325 & I.0I (0.99-I.02) & 0.389 \\
\hline South & $1.10(1.09-1.12)$ & $<0.001$ & $1.10(1.08-1.12)$ & $<0.001$ \\
\hline West & $1.02(1.01-1.04)$ & 0.004 & $1.02(1.00-1.04)$ & 0.019 \\
\hline Unknown & $1.06(1.01-1.12)$ & 0.020 & $1.08(1.02-1.13)$ & 0.028 \\
\hline
\end{tabular}

Notes: aln this analysis, patients who discontinue branded desvenlafaxine will be censored at the end of their branded desvenlafaxine use. All patients were censored at the end of follow-up.

Abbreviations: SSRIs, selective serotonin reuptake inhibitors; SNRIs, selective norepinephrine reuptake inhibitors; POS, point of service; HMO, Health Maintenance Organization; PPO, preferred provider organization; HR, hazard ratio; MDD, major depressive disorder.

or sertraline after initial treatment failure with citalopram, indicating that switching after the initial treatment might be beneficial, especially in recurrent and unresponsive cases. ${ }^{19}$ In some patients with MDD, combination therapy might be prescribed to the unresponsive patients. It is important for clinicians to evaluate the patients' symptoms and prior history to determine an optimal treatment regimen.

In our study, the concomitant use of antidepressant medications was slightly higher in patients treated with branded desvenlafaxine compared with the other branded 
Table 5 Concomitant antidepressant drug therapy

\begin{tabular}{|c|c|c|c|}
\hline & \multicolumn{3}{|l|}{ Patients, n (\%) } \\
\hline & $\begin{array}{l}\text { Branded desvenlafaxine } \\
(\mathrm{N}=14,379)\end{array}$ & $\begin{array}{l}\text { Other branded SSRIs/SNRIs } \\
(\mathrm{N}=50,937)\end{array}$ & $\begin{array}{l}\text { Generic SSRIs/SNRIs } \\
(\mathrm{N}=\mathbf{2 0 8}, 198)\end{array}$ \\
\hline Any concomitant medications & $6,294(43.8)$ & $20,282(39.8)$ & $75,768(36.4)$ \\
\hline \multicolumn{4}{|l|}{ Concomitant medications $^{\mathrm{a}}$} \\
\hline Atypical antidepressant only & $2,333(37.1)$ & $8,230(40.6)$ & $36,846(48.6)$ \\
\hline Antipsychotic only & $1,162(18.5)$ & $2,796(\mid 3.8)$ & $9,026(11.9)$ \\
\hline Atypical antidepressant and antipsychotic & $677(10.8)$ & $\mathrm{I}, 743(8.6)$ & $6,287(8.3)$ \\
\hline Generic SSRI only & $450(7.1)$ & I,737 (8.6) & $3,792(5.0)$ \\
\hline Tricyclic antidepressant only & $292(4.6)$ & $\mathrm{I}, \mathrm{I} 55(5.7)$ & $4,156(5.5)$ \\
\hline Branded SNRI only & $89(1.4)$ & $206(1.0)$ & I,924 (2.5) \\
\hline Generic SNRI only & $84(1.3)$ & $452(2.2)$ & $\mathrm{I}, 347(\mathrm{I} .8)$ \\
\hline Branded SSRI only ${ }^{\mathrm{b}}$ & $90(1.4)$ & $247(1.2)$ & $764(1.0)$ \\
\hline Branded desvenlafaxine only & $0(0.0)$ & $|3|(0.6)$ & $421(0.6)$ \\
\hline MAOI only & $\mathrm{I}(0.0)$ & $\mathrm{I}(0.0)$ & $0(0.0)$ \\
\hline \multicolumn{4}{|l|}{ Other antidepressant combinations } \\
\hline Two classes & $827(13.1)$ & $2,686(\mid 3.2)$ & $8,526(11.3)$ \\
\hline Three classes & $258(4.1)$ & $785(3.9)$ & $2,388(3.2)$ \\
\hline$\geq 4$ classes & $31(0.5)$ & II $3(0.6)$ & $291(0.4)$ \\
\hline
\end{tabular}

Notes: aDenominator for percentage is patients with any concomitant use. ${ }^{b}$ Branded SSRIs include the study drugs Lexapro (escitalopram) and nonstudy SSRI drugs Celexa (citalopram hydrobromide), Luvox (fluvoxamine maleate), Zoloft (sertraline hydrochloride), Prozac (fluoxetine), Sarafem (fluoxetine hydrochloride), Paxil (paroxetine), Brisdelle (paroxetine), and Pexeva (paroxetine).

Abbreviations: SSRI, selective serotonin reuptake inhibitor; SNRI, serotonin-norepinephrine reuptake inhibitor; MAOI, monoamino oxidase inhibitor.

and generic SSRIs and SNRIs. This might be explained by the fact that patients were on branded desvenlafaxine for a longer time compared with other categories. In addition, we found a higher overall use of concomitant antidepressant therapies in our cohort compared with the previously published estimates. ${ }^{8}$ We also found that antipsychotics were more often concomitantly used alongside branded desvenlafaxine compared with other (branded or generic) SNRIs/ SSRIs. The high concomitant behavior might be influenced by desvenlafaxine's tolerability toward other drugs. ${ }^{20}$

Information on the use of concomitant antidepressant medications and factors influencing patterns of concomitant antidepressant drug use among MDD patients is somewhat limited. Based on the results from a retrospective claims data analyses, antidepressant dosage and seeing multiple prescribers may lead to higher use of concomitant antidepressant therapy. ${ }^{21}$ Other factors that contribute to the use of concomitant antidepressant medications in MDD patients are increased burden of illness, comorbidities, and lower drug effectiveness. ${ }^{22}$ Concomitant use of medications in MDD was also related to white race, having insurance, anxiety disorder, and PTSD, ${ }^{22}$ which were well represented in our study cohort.

Overall persistence to branded desvenlafaxine in our study was higher than estimates found in the existing literature for other SSRIs/SNRIs. ${ }^{23,24}$ In another retrospective study using IMS LifeLink claims data (2003-2004), the persistence to SSRIs was $37.6 \%$ at 3 months and $18.9 \%$ at 6 months. ${ }^{25}$ In a study by Lin et a ${ }^{26}$ based on medical records from two primary care clinics, at least $40 \%$ of patients discontinued fluoxetine, TCA, and trazodone therapy by the end of 3 months. However, in a review of studies in psychiatric populations over the past 10 years, the average rate of patients (based on up to 6-month assessment) not taking the medication as indicated was $52 \%$, which is more aligned with our study estimates. ${ }^{27}$

All MDD medications including desvenlafaxine have certain benefits and risks. When treating patients with MDD, it is important to look at not only clinical efficacy but also the safety profile of available antidepressant therapies. The adverse event profiles and potential for drug interactions with antidepressant therapies vary with the drug therapy (eg, desvenlafaxine is associated with decreased sex drive and a rare likelihood of serotonin syndrome, sertraline for risk of diarrhea, vomiting and nausea for venlafaxine users, and paroxetine is responsible for sexual dysfunction). ${ }^{28}$ However, results from two recently published reviews found slight but nonsignificant differences among individual SSRIs/SNRIs with regard to efficacy and safety. ${ }^{29,30}$ The American College of Physicians recommend that clinicians monitor all patients initiated on antidepressant therapy for the first 2 weeks to evaluate response and development of adverse events and modify the therapy after at least $6-8$ weeks of treatment as needed. ${ }^{17}$ 
Limitations of this study are typical of database analyses and include the reliance on diagnosis, coding, and lack of clinical details from patient's medical charts. Claims data do not record the true medication behaviors of patients and we assume that prescription claims reflect how patients take medications. Condition severity and patient health status were not captured and adjusted, which might confound the results. Establishing the relationship between MDD and antidepressant use is challenging, as MDD patients might use antidepressants for other associated indications. Outcomes were not evaluated in mutually exclusive groups; thus, the patient outcomes may not be entirely attributed to the index drug. Furthermore, brand to generic switching or vice versa was not examined, and further investigation of switching patterns is warranted in future studies. Although the focus of this study was to compare persistence across broader drug categories, a sensitivity analysis to compare persistence of individual drugs was also performed to gain a deeper understanding of medication usage and behavior. Clearly, the persistence pattern differences observed between the brand and generic drug versions suggest the influence of additional factors on persistence. These factors are hard to identify in a retrospective study. The impact of clinical characteristics and subgroups, formulary tier placement, required substitution policies, copays, and patient incentive, and assistance programs are all likely to impact persistence. ${ }^{31-33}$

Despite these limitations, several strengths of this study can be mentioned. Truven MarketScan is a large database and contains patient data from all regions of the US and a number of managed care plans. The large sample size analyzed provides robustness to the real-world evidence and helps make the results more generalizable. Data provided by this study can serve as a baseline for identifying areas of further exploration relating to MDD treatment patterns, comparative persistence, and health care resource utilization across antidepressant medications. The analysis also serves to build awareness regarding how treatments for MDD are prescribed, which may aid clinicians in making treatment and formulary decisions. Further investigation of results with matched samples and stratification by individual drug/ therapeutic class is warranted.

\section{Conclusion}

Our study was the first to explore the treatment persistence of branded desvenlafaxine along with other branded and generic SSRI or SNRI drugs. Despite the limitations of retrospective claim data analyses, our results provide valuable insight on SSRI and SNRI drug use patterns and concomitant drug use in MDD patients. Compared with patients on branded desvenlafaxine, patients receiving other branded SSRIs or SNRIs were $31 \%$ and generic SSRIs or SNRIs were $11 \%$ more likely to discontinue antidepressant therapy. Further studies are needed to identify underlying factors, such as patient copayment amounts, that may be associated with persistence trends in patients treated with MDD drugs.

\section{Acknowledgments}

The study was designed and data were analyzed by Pharmerit International. The authors would like to thank other Pharmerit project team members for their contributions to the research, interpretation, and critical review: Xin Gao, $\mathrm{PhD}$; Jennifer Stephens, PharmD; and Lavanya Sudharshan, MS. The study was supported by Pfizer, Inc.

\section{Disclosure}

Caitlyn Solem, Yin Wan, and Chinmay Deshpande are employees of Pharmerit International who were paid consultants to Pfizer in connection with the development of this manuscript. Ahmed Shelbaya, Elizabeth Pappadopulos, and Jose Alvir are employees of Pfizer, Inc. The authors report no other conflicts of interest in this work.

\section{References}

1. Administration SAaMHS. Results from the 2013 National Survey on Drug Use and Health: Mental Health Findings, NSDUH Series H-49, HHS Publication No (SMA) 14-4887. Rockville, MD: 2013.

2. Ferrari AJ, Charlson FJ, Norman RE, et al. The epidemiological modelling of major depressive disorder: application for the Global Burden of Disease Study 2010. PLoS One. 2013;8(7):e69637.

3. Andrade L, Caraveo-Anduaga JJ, Berglund P, et al. The epidemiology of major depressive episodes: results from the International Consortium of Psychiatric Epidemiology (ICPE) Surveys. Int J Methods Psychiatr Res. 2003;12(1):3-21.

4. Greenberg PE, Kessler RC, Birnbaum HG, et al. The economic burden of depression in the United States: how did it change between 1990 and 2000? J Clin Psychiatry. 2003;64(12):1465-1475.

5. Kocsis JH, Gelenberg AJ, Rothbaum B, et al. Chronic forms of major depression are still undertreated in the 21 st century: systematic assessment of 801 patients presenting for treatment. J Affect Disord. 2008; 110(1):55-61.

6. American Psychiatric Association. Practice Guidleine for the Treatment of Patients With Major Depressive Disorder. Third ed. Arlington, VA: American Psychiatric Association; 2010.

7. Koenig AM, Thase ME. First-line pharmacotherapies for depression-what is the best choice. Pol Arch Med Wewn. 2009;119(7-8):478-486.

8. Rascati K. Drug utilization review of concomitant use of specific serotonin reuptake inhibitors or clomipramine with antianxiety/sleep medications. Clin Ther. 1995;17(4):786-790.

9. Papakostas GI, Shelton RC, Smith J, Fava M. Augmentation of antidepressants with atypical antipsychotic medications for treatmentresistant major depressive disorder: a meta-analysis. J Clin Psychiatry. 2007;68(6):826-831.

10. DiMatteo MR, Lepper HS, Croghan TW. Depression is a risk factor for noncompliance with medical treatment: meta-analysis of the effects of anxiety and depression on patient adherence. Arch Intern Med. 2000; 160(14):2101-2107. 
11. Wade AG, Häring J. A review of the costs associated with depression and treatment noncompliance: the potential benefits of online support. Int Clin Psychopharmacol. 2010;25(5):288-296.

12. Nantz E, Liu-Seifert H, Skljarevski V. Predictors of premature discontinuation of treatment in multiple disease states. Patient Prefer Adherence. 2009;3:31.

13. Ruhe HG, Huyser J, Swinkels JA, Schene AH. Switching antidepressants after a first selective serotonin reuptake inhibitor in major depressive disorder: a systematic review. J Clin Psychiatry. 2006;67(12): 1836-1855.

14. Spina E, Trifirò G, Caraci F. Clinically significant drug interactions with newer antidepressants. CNS Drugs. 2012;26(1):39-67.

15. American Psychiatric Association. Diagnostic and Statistical Manual of Mental Disorders (DSM-5®). Arlington, VA: American Psychiatric Pub; 2013.

16. Trivedi MH, Rush AJ, Wisniewski SR, et al. Evaluation of outcomes with citalopram for depression using measurement-based care in STAR* D: implications for clinical practice. American Journal of Psychiatry.2014; 163(1):28-40.

17. Qaseem A, Snow V, Denberg TD, Forciea MA, Owens DK. Using second-generation antidepressants to treat depressive disorders: a clinical practice guideline from the American College of Physicians. Ann Intern Med. 2008;149(10):725-733.

18. Melfi CA, Chawla AJ, Croghan TW, Hanna MP, Kennedy S, Sredl K. The effects of adherence to antidepressant treatment guidelines on relapse and recurrence of depression. Arch Gen Psychiatry. 1998;55(12): $1128-1132$.

19. Rush AJ, Trivedi MH, Wisniewski SR, et al. Acute and longer-term outcomes in depressed outpatients requiring one or several treatment steps: a STAR* D report. Am J Psychiatry. 2006;163(11): 1905-1917.

20. Nichols A, Lubaczewski S, Liang Y, Matschke K, Braley G. An openlabel evaluation of the effect of coadministering desvenlafaxine $100 \mathrm{mg}$ on the pharmacokinetics of aripiprazole in healthy subjects. J Bioequiv Availab. 2013;5:253-259.

21. Kotzan JA, Maclean R, Wade W, et al. Prevalence and patterns of concomitant use of selective serotonin reuptake inhibitors and other antidepressants in a high-cost polypharmacy cohort. Clin Ther. 2002;24(2): $237-248$.
22. Shelton RC, Hollon SD, Wisniewski SR, et al. Factors associated with concomitant psychotropic drug use in the treatment of major depression: a STAR* D Report. CNS Spectr. 2009;14(09):487-498.

23. Wang J, Liu X, Mullins CD. Treatment adherence and persistence with duloxetine, venlafaxine XR, and escitalopram among patients with major depressive disorder and chronic pain-related diseases. Curr Med Res Opin. 2011;27(7):1303-1313.

24. Mullins CD, Shaya FT, Meng F, Wang J, Harrison D. Persistence, switching, and discontinuation rates among patients receiving sertraline, paroxetine, and citalopram. Pharmacotherapy. 2005;25(5):660-667.

25. Ereshefsky L, Saragoussi D, Despiégel N, Hansen K, François C, Maman K. The 6-month persistence on SSRIs and associated economic burden. J Med Econ. 2010;13(3):527-536.

26. Lin EH, Von Korff M, Katon W, et al. The role of the primary care physician in patients' adherence to antidepressant therapy. Med Care. 1995;33(1):67-74.

27. Sansone RA, Sansone LA. Antidepressant adherence: are patients taking their medications? Innov Clin Neurosci. 2012;9(5-6):41-46.

28. Dean L [webpage on the Internet]. Comparing Antidepressants PubMed Clinical $Q \& A$; 2011. Available from: http://www.ncbi.nlm.nih.gov/ pubmedhealth/PMH0004912/. Accessed August 18, 2016

29. Jia Y, Zhu H, Leung S-W. Comparative efficacy of selective serotonin reuptake inhibitors (SSRI) in treating major depressive disorder: a protocol for network meta-analysis of randomised controlled trials. BMJ Open. 2016;6(6):e010142.

30. Gartlehner G, Thaler K, Hill S, Hansen RA. How should primary care doctors select which antidepressants to administer? Curr Psychiatry Rep. 2012;14(4):360-369.

31. Assayag J, Forget A, Kettani F-Z, Beauchesne M-F, Moisan J, Blais L. The impact of the type of insurance plan on adherence and persistence with antidepressants: a matched cohort study. Can J Psychiatry. 2013; 58(4):233-239.

32. Barron J, Wahl P, Fisher M, Plauschinat C. Effect of prescription copayments on adherence and treatment failure with oral antidiabetic medications. P T. 2008;33(9):532-553.

33. Zeng F, Patel B, Andrews L, Frech-Tamas F, Rudolph A. Adherence and persistence of single-pill $\mathrm{ARB} / \mathrm{CCB}$ combination therapy compared to multiple-pill ARB/CCB regimens. Curr Med Res Opin. 2010;26(12): 2877-2887.
Neuropsychiatric Disease and Treatment

\section{Publish your work in this journal}

Neuropsychiatric Disease and Treatment is an international, peerreviewed journal of clinical therapeutics and pharmacology focusing on concise rapid reporting of clinical or pre-clinical studies on a range of neuropsychiatric and neurological disorders. This journal is indexed on PubMed Central, the 'PsycINFO' database and CAS,

\section{Dovepress}

and is the official journal of The International Neuropsychiatric Association (INA). The manuscript management system is completely online and includes a very quick and fair peer-review system, which is all easy to use. Visit http://www.dovepress.com/testimonials.php to read real quotes from published authors. 